\section{Painting New Lines: Maximizing Color Difference in Metro Maps}

\section{Simone F. Griffioen AND Arthemy V. Kiselev}

This column is a place for those bits of contagious mathematics that travel from person to person in the community, because they are so elegant, surprising, or appealing that one has an urge to pass them on. Contributions are most welcome.

\footnotetext{
> Submissions should be uploaded to http://tmin.edmgr.com or sent directly to Sergei Tabachnikov, tabachni@math.psu.edu
}

\begin{abstract}
"Have you seen the new terminal on the green?"
"The red is overcrowded every morning at this time."

"I live at the very end of orange, still OK with me: the escalator brings me right into the office."
\end{abstract}

"I have to change to purple here. See you tomorrow!"

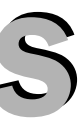
ure, it is all about metro. The subway. The metropoli tan underground railway system. Each line has its own color and each color is the name of a line. A problem arises when another line is built: the old lines must keep their colors, whereas the new line should have a color that looks maximally different from the old ones. It becomes even more complicated if there are two or more new lines in a subway. Not only must the new colors be unlike the old ones, but also they must differ from each other as much as possible.

In this article we discuss a mathematical strategy to choose these colors. By the way, what new colors would you suggest for the real metro map in Figure 1 ? $^{1}$ For our purposes this map is ideal: not only are there 14 colors in it, but also there will be several new lines built in that subway in the near future (according to [2], and the planned extensions are in Figure 6). Let us use this map to illustrate our strategy-yet the reader can apply the techniques to whatever other map he or she likes (we invite the reader to analyse the London metro map in Figure $2^{2}$ ).

\section{Measuring the Color Difference}

To maximize color differences-here, in metro maps-we need a way to measure them and argue about colors objectively. For this we describe them as tuples of numbers by using some coordinate system (or color space). For example, the red, green, and blue (RGB) color space is well known: in it every color is encoded by its RGB components. Unfortunately, in the RGB space the Euclidean distance is not proportional to the visible distance that people perceive between colors. That is, the RGB space is not perceptually uniform-which we would like.

In 1931, the Commission Internationale de l'Eclairage (CIE) did measurements on the vision of hundreds of humans. From those data, several color spaces were created to make communication about colors easy and unambiguous. In 1976, the CIE recommended the use of

\footnotetext{
1 Opened in 1935 with 11 kilometres of lines and 13 stations, the Moscow metro has 196 stations nowadays and the total route length of its lines is at least $327.5 \mathrm{~km}$ [5]. It is one of the world's most heavily used metro systems.

${ }^{2}$ The London underground system serves 270 stations and has 402 kilometres of track. Opened in 1863, it is the world's oldest underground railway network and is one of the largest [14].
} 


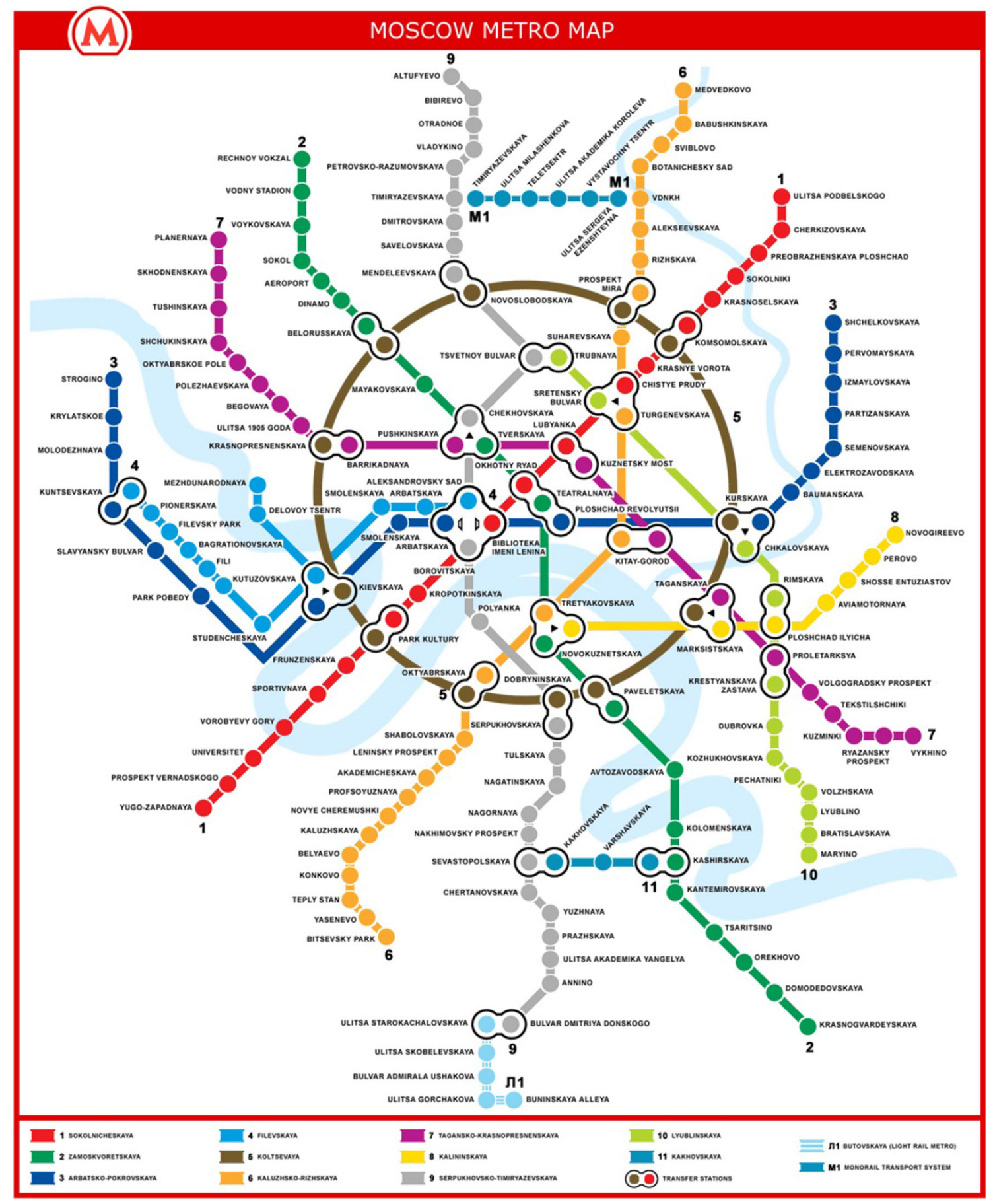

Figure I. It is likely that you have already recognised the Moscow metro here.

two approximately perceptually uniform color spaces and color-difference formulas. These spaces have become known by their officially recommended abbreviations CIELAB and CIELUV. In the former, a color is described by three coordinates: $L$ (lightness), $a$ (red-green scale), and $b$ (yellow-blue scale). ${ }^{3}$ We will use this space to describe the colors of the Moscow metro map in terms of numbers: their
CIELAB coordinates can be found in Table 1; Figure 3 shows a stereographical plot of the colors of the Moscow metro in the CIELAB space.

The CIELAB space itself is not bounded; however, based on what one wants to measure, only part of it matters in practice (for example, only the colors that a printer can print or only the colors the human eye can see). Such a

\footnotetext{
${ }^{3}$ The L-scale runs from 0 to 100; the parameters $a$ and $b$ typically have values in the range -150 to 150 . In this article we will round the coordinates of the colors to integers. The human eye can discriminate up to ten million colors, so it can distinguish even finer differences than integer steps.
} 


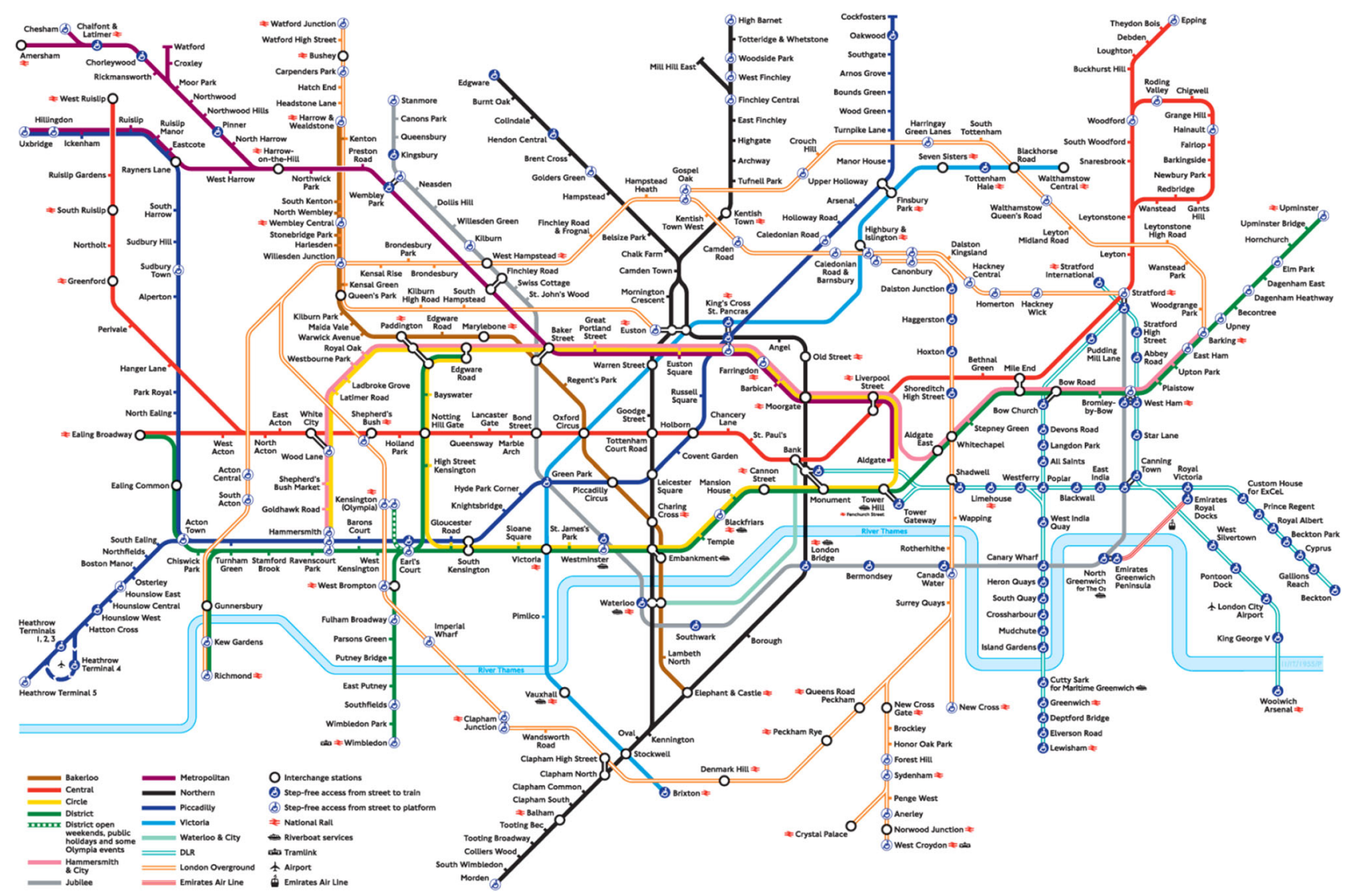

Figure 2. The map of the London metro.

subset of colors is called a color gamut. The gamut of Adobe 98 in the CIELAB space can be seen in Figure 4. To keep the problem simple, we approximate this gamut by a polyhedron. Because the CIELAB space was supposed to be almost perceptually uniform, the Euclidean distance gives the CIE 1976 color difference formula (CIE76)

$$
\Delta E_{a b}=\sqrt{\left(L_{1}-L_{2}\right)^{2}+\left(a_{1}-a_{2}\right)^{2}+\left(b_{1}-b_{2}\right)^{2}} .
$$

In fact, the CIELAB space is not really uniform. In particular, at high values of $a$ and $b$, the far-too-simple CIE76 formula values color differences too strongly compared to the experimental results on color perception. This is why several new color-difference formulas were proposed, of which the newest is the CIEDE2000 formula ${ }^{4}$ (or $\Delta E_{00}$ ).

\section{Mathematical Formulation of the Problem}

Given the CIELAB space equipped with the Euclidean metric-or even better, the CIEDE2000 color difference formula-and the color gamut $\Gamma$ (which we chose to be a polyhedron), we consider a set of existing colors $P=$ $\left\{p_{1}, \ldots, p_{k}\right\}$ within this gamut, represented by their CIELAB coordinates (e.g., see Table 1). If we want to add only one new line, this leads to the following maximin problem:

Problem 1 (one new color). How can we find an $x \in \Gamma$ such that

$$
\min _{p_{i} \in P}\left\|x-p_{i}\right\| \rightarrow \max _{x \in \Gamma} ?
$$

That is, the minimal distance from the new color $x$ to the existing ones must be as large as possible, provided that the color $x$ stays inside or on the boundary of the gamut $\Gamma$.

If we want to add several new lines, each having its own new color, the maximin problem becomes this:

Problem 2 ( $m$ new colors). How can we find a collection of $m$ points $X=\left\{x_{1}, \ldots, x_{m}\right\}$ such that

$$
\min _{\substack{x_{i}, x_{j} \in X \\ i \neq j \\ p_{k} \in P}}\left\{\left\|x_{i}-x_{j}\right\|,\left\|x_{i}-p_{k}\right\|\right\} \rightarrow \max _{X \subset \Gamma} ?
$$

\footnotetext{
${ }^{4}$ In the CIEDE2000 distance, some weighting coefficients have to be chosen. We use the ratio $k_{L}^{-1}: k_{c}^{-1}: k_{h}^{-1}=\frac{1}{2}: 1: 1$. This means that we consider the difference in chroma $c=\sqrt{a^{2}+b^{2}}$ and hue $b=\arctan (b / a)$ to be more important than the difference in lightness $L$. For more information about this color difference formula, see [4].
} 


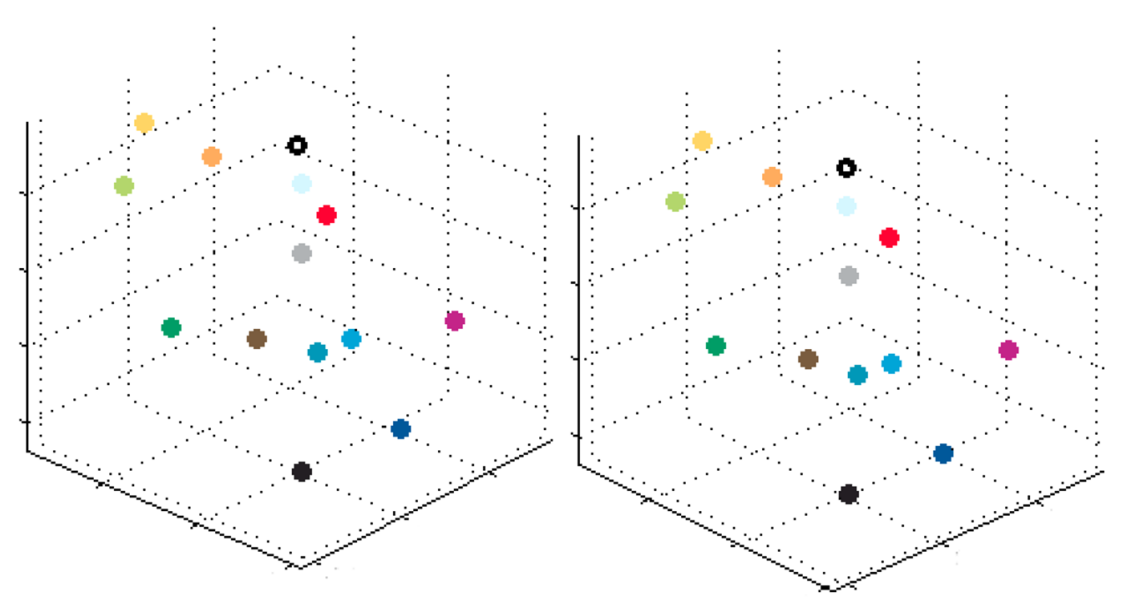

Figure 3. A stereographic image of the colors of the Moscow metro map at their location in the CIELAB space. The $L$-axis is the vertical axis, the $a$-axis runs from southwest to northeast, and the $b$-axis from southeast to northwest. (To see $a$ 3-dimensional picture, view the left picture with your left eye and the right picture with your right eye at the same time.)

\begin{tabular}{|c|c|c|c|c|c|}
\hline Line & Color & $L$ & $a$ & $\boldsymbol{b}$ & $\min \Delta E_{00}$ \\
\hline 1 & - Red & 52 & 74 & 53 & 29.1 \\
\hline 2 & - Ocean green & 56 & -45 & 26 & 21.7 \\
\hline 3 & - Cobalt & 35 & 7 & -43 & 19.4 \\
\hline 4 & Sky blue & 61 & -16 & -42 & 6.3 \\
\hline 5 & - Olive brown & 41 & 6 & 27 & 20.4 \\
\hline 6 & Peach & 76 & 24 & 67 & 14.5 \\
\hline 7 & - Pinkish purple & 43 & 64 & -24 & 28.3 \\
\hline 8 & Light mustard & 86 & 4 & 85 & 14.5 \\
\hline 9 & Light grey & 71 & 0 & -2 & 9.4 \\
\hline 10 & Greenish yellow & 80 & -26 & 68 & 17.8 \\
\hline 11 & - Turquoise blue & 56 & -21 & -29 & 6.3 \\
\hline Water & Pale blue & 93 & -8 & -9 & 11.3 \\
\hline Background & O White & 100 & 0 & 0 & 9.4 \\
\hline Text & - Black & 13 & 2 & 0 & 20.4 \\
\hline
\end{tabular}

That is, we maximize both the minimal distance in between the new colors $x_{1}, \ldots, x_{m}$ and their minimal distance to the old colors. Alternatively, we could add the new colors $x_{1}, \ldots, x_{m}$ one by one, using a method for solving Problem 1. However this does not always yield the optimal result. 5

\section{The Voronoi Diagram Method}

Let us consider Problem 1. Because the CIELAB space is continuous (at least in principle), it is impossible to "try all points" in the gamut to find the one with the maximal minimal distance. Therefore it would be much easier to have only a finite number of candidate points to check. This can be achieved by using Voronoi diagrams.

Definition 1. Let $P=\left\{p_{1}, \ldots, p_{k}\right\} \subset \mathbb{R}^{3}$ be a finite set (e.g., of old colors) such that $p_{i} \neq p_{j}$ for $i \neq j$. The Vorono $i$ polybedron of a point $p_{i}$ is the region

$$
V\left(p_{i}\right):=\left\{x \in \mathbb{R}^{3}:\left\|x-p_{i}\right\| \leq\left\|x-p_{j}\right\| \text { for all } j \neq i\right\} .
$$

The set $\mathcal{V}(P)=\left\{V\left(p_{i}\right), \ldots, V\left(p_{k}\right)\right\}$ is the (3-dimensional) Voronoi diagram generated by the set of points $P$.

It is easily shown that the sought-for point $x$ maximizing the minimal distance in Problem 1 belongs to the intersection of

either: three Voronoi planes, that is, three faces of Voronoi polyhedra,

or: two Voronoi planes and a boundary plane of the gamut,

or: a Voronoi plane and two boundary planes,

or: three boundary planes.

This means that as soon as these candidate points are calculated, ${ }^{6}$ it only remains to compare their minimal distances to the existing points $p_{i} \in P$, and then to take the one whose distance is maximal; in fact, the resulting set is finite and reasonably small. This is the CIE76 Voronoi method of solving Problem 1 with respect to the Euclidean distance. In Table 2 we list the five new colors that we obtained, one by one, for the Moscow metro by using this method.

It is readily seen that the Voronoi method would provide the global optimal solution to maximin Problem 1-if there

\footnotetext{
${ }^{5}$ Metro lines are built one after another, so choosing new colors one by one is quite logical. However, sometimes we know in advance that several new lines are being built.

6 This can be done by elementary linear algebra, that is, by calculating bisectors and intersections of planes. In Figure 5, a 2-dimensional example of a Voronoi diagram can be found.
} 


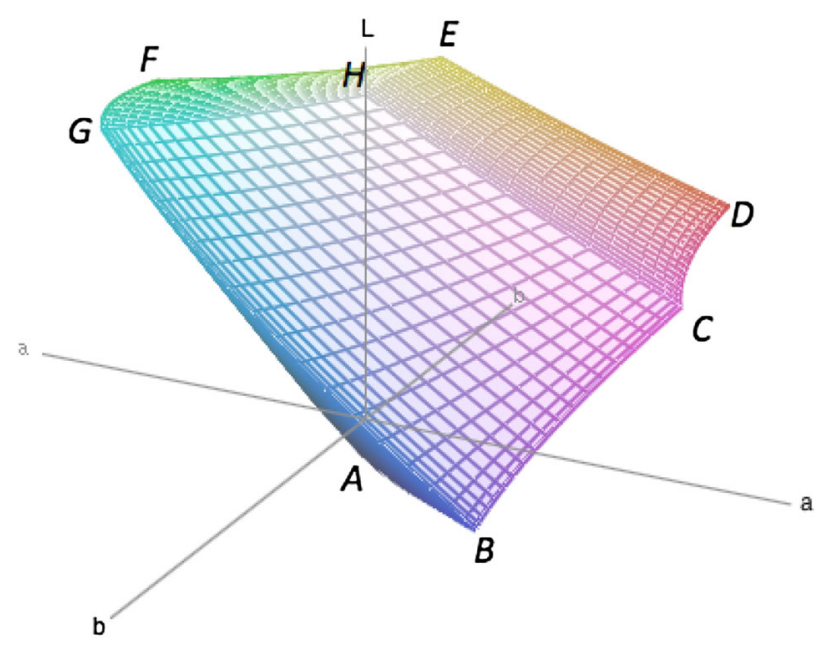

Figure 4. The gamut of Adobe 98 in the CIELAB space. We approximate this gamut by a polyhedron by taking the corner points of this polyhedron close to the points $A, \ldots, H$ in the figure.

were a perceptually uniform color space. This is not yet the case nowadays; therefore let us study how better estimates can be obtained.

Because the CIEDE2000 distance is much more accurate than the Euclidean distance, we can improve our method by selecting the optimal point from the candidate points by comparing the $\Delta E_{00}$ distances instead of the Euclidean distances $\Delta E_{a b}$. This is the combined CIE76-CIEDE200O Voronoi method. Of course, this method does not solve Problem 1 with respect to $\Delta E_{00}$ alone, because the candidate points are found by using $\Delta E_{a b}$. However it is a fast method to obtain colors by using the CIEDE2000 distance. The five new colors obtained, one by one, for the Moscow metro by this method are given in Table $3 .^{7}$ We see that this gives us much better solutions with respect to the CIEDE2000 distance than the Voronoi CIE76 method: indeed, the $\Delta E_{00}$ distance drops at a much slower rate.

\section{The Simplex Method}

We can also solve Problem 2 with respect to the Euclidean distance by using the simplex method. Let us consider here the case when the number of colors $m$ is two, which gives us the following function to maximize,

$$
M(x, y)=\min _{\substack{p_{i} \in P \\ x, y \in \Gamma}}\left\{\left\|x-p_{i}\right\|,\left\|y-p_{i}\right\|,\|x-y\|\right\} .
$$

We have $(x, y) \in \Gamma \times \Gamma \subset \mathbb{R}^{6}$. Let us rewrite our problem: first, put one extra parameter $x_{0}$ and the two vectors $x, y$ together in $\tilde{x}=\left(x_{0}, x, y\right) \in \mathbb{R}^{7}$, and now maximize the function $M_{0}(\tilde{x})=x_{0}$ over all $\tilde{x} \in \mathbb{R} \times \Gamma \times \Gamma$ satisfying the constraints

$$
\left\|x-p_{i}\right\|-x_{0} \geq 0 \quad \text { for } \text { each } p_{i} \in P,
$$

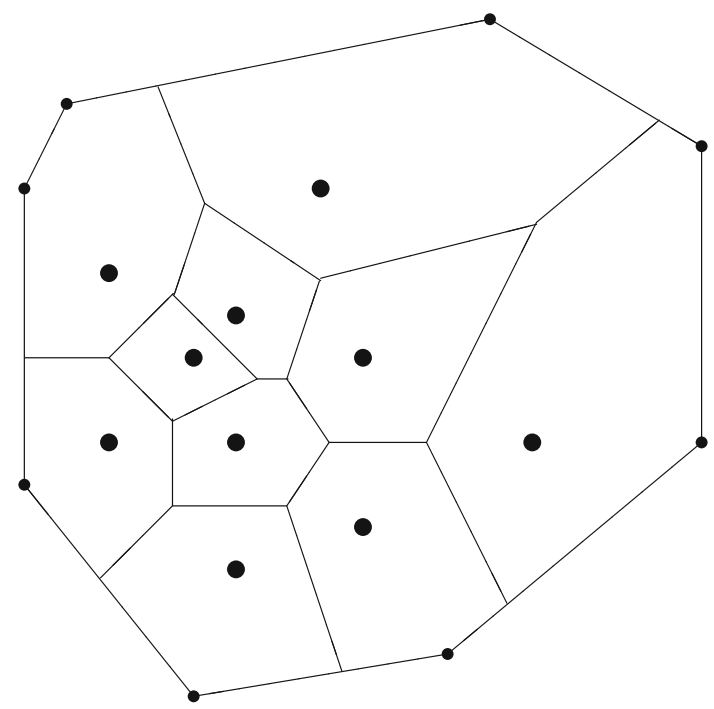

Figure 5. A 2-dimensional Voronoi diagram. Note that all edges of the Voronoi polygons are parts of the bisectors of lines connecting two points in the diagram.

Table 2. Colors we find one by one for the first five new lines in the Moscow metro by using the CIE76 Voronoi method, that is, by solving Problem 1 with respect to the Euclidean distance. The minimal distances that we indicate here take in account the points that came earlier into the table. Note that the minimal $\Delta \mathrm{E}_{00}$ distance is then not necessarily decreasing.

\begin{tabular}{lclllr}
\hline Color & $\min \Delta \mathbf{E}_{\mathrm{ab}}$ & $\min \Delta \mathbf{E}_{00}$ & \multicolumn{1}{l}{$\boldsymbol{L}$} & \multicolumn{1}{c}{$\boldsymbol{a}$} & \multicolumn{1}{c}{$\boldsymbol{b}$} \\
\hline - Bright green & 114.4 & 22.0 & 83 & -138 & 91 \\
- Blue & 87.0 & 19.8 & 33 & 80 & -109 \\
- Sea green & 67.7 & 13.1 & 85 & -106 & 31 \\
- Cyan & 65.3 & 21.9 & 87 & -78 & -21 \\
- Bright pink & 56.1 & 14.4 & 60 & 100 & -64 \\
\hline
\end{tabular}

$$
\begin{aligned}
& \left\|y-p_{j}\right\|-x_{0} \geq 0 \quad \text { for each } p_{j} \in P, \\
& \|x-y\|-x_{0} \geq 0
\end{aligned}
$$

Of course, more constraints on $(x, y)$ are used to delimit the set $\Gamma \times \Gamma$. Now we use the simplex method to maximize our function.

The gamut's boundary gives the linear constraints, and the nonlinear constraint functions are the three inequalities (1). Because the simplex method gives us only a local optimum, we apply it for a million random initial points $\left(x_{0}, x, y\right) \in \mathbb{R}^{7}$ (with $x_{0} \in[0,20]$ and $(x, y) \in \Gamma \times \Gamma$ ). These are local optima with respect to the $\Delta E_{a b}$ distance; let us now choose the one with maximal $\Delta E_{00}$ distance. This way, we obtain the two colors

$$
\text { Bright aqua }(84,-65,-12) \text { and Baby pink } \bullet(86,35,7) \text {. }
$$

Their combined minimal CIEDE2000 distance to the old colors and each other is 22.7 . This is more than the minimal

\footnotetext{
7 The minimal CIEDE2000 distances do not always decrease at each step; this is because the candidate points are selected by using the Euclidean distance, which does not necessarily give an optimum with respect to the CIEDE2000 distance. Therefore it is possible that, after adding a new color, one of the new candidate points is an even better solution than the point added before.
} 
distance that was obtained after two steps of the one-byone CIE76-CIEDE2000 Voronoi method. So this gives us a better result for solving Problem 2. However, this method is quite time-consuming (as $m$ increases, the simplex method

Table 3. Colors we find one by one for the first five new lines using the combined CIE76-CIEDE2000 Voronoi method. Note that the distances here are the CIEDE2000 distances, and they drop at a much slower rate than in Table 2.

\begin{tabular}{lllrr}
\hline Color & $\min \Delta \mathbf{E}_{\mathbf{0 0}}$ & $\boldsymbol{L}$ & \multicolumn{1}{c}{$\boldsymbol{a}$} & \multicolumn{1}{c}{$\boldsymbol{b}$} \\
\hline Cyan & 23.9 & 87 & -78 & -21 \\
Bright green & 22.0 & 83 & -138 & 91 \\
Blue & 19.8 & 33 & 80 & -109 \\
Pale lavender & 19.7 & 86 & 23 & -22 \\
Rose pink & 19.8 & 78 & 59 & 12 \\
\hline
\end{tabular}

will need even more time), so the much faster Voronoi method is a good alternative.

\section{Conclusion and Discussion}

We conclude that the Voronoi diagram method (and its improvement by using both the CIE76 and CIEDE2000 distance functions) for adding new colors one by one gives solutions that are comparable with the ones produced by the nonsmooth optimization method for finding two or more new colors at once. This is true for the map of the Moscow metro under study. Other solution schemes are of course possible, for example the Monte-Carlo method: testpoints $x_{i} \in \Gamma$ for the new color(s) are generated within the gamut at random, and the new color-or a tuple of new colors-is the maximin optimum over a sufficiently large number of such attempts. The ballistic method is realized as follows: first, suppose that each old color $p_{i} \in \Gamma$ and every

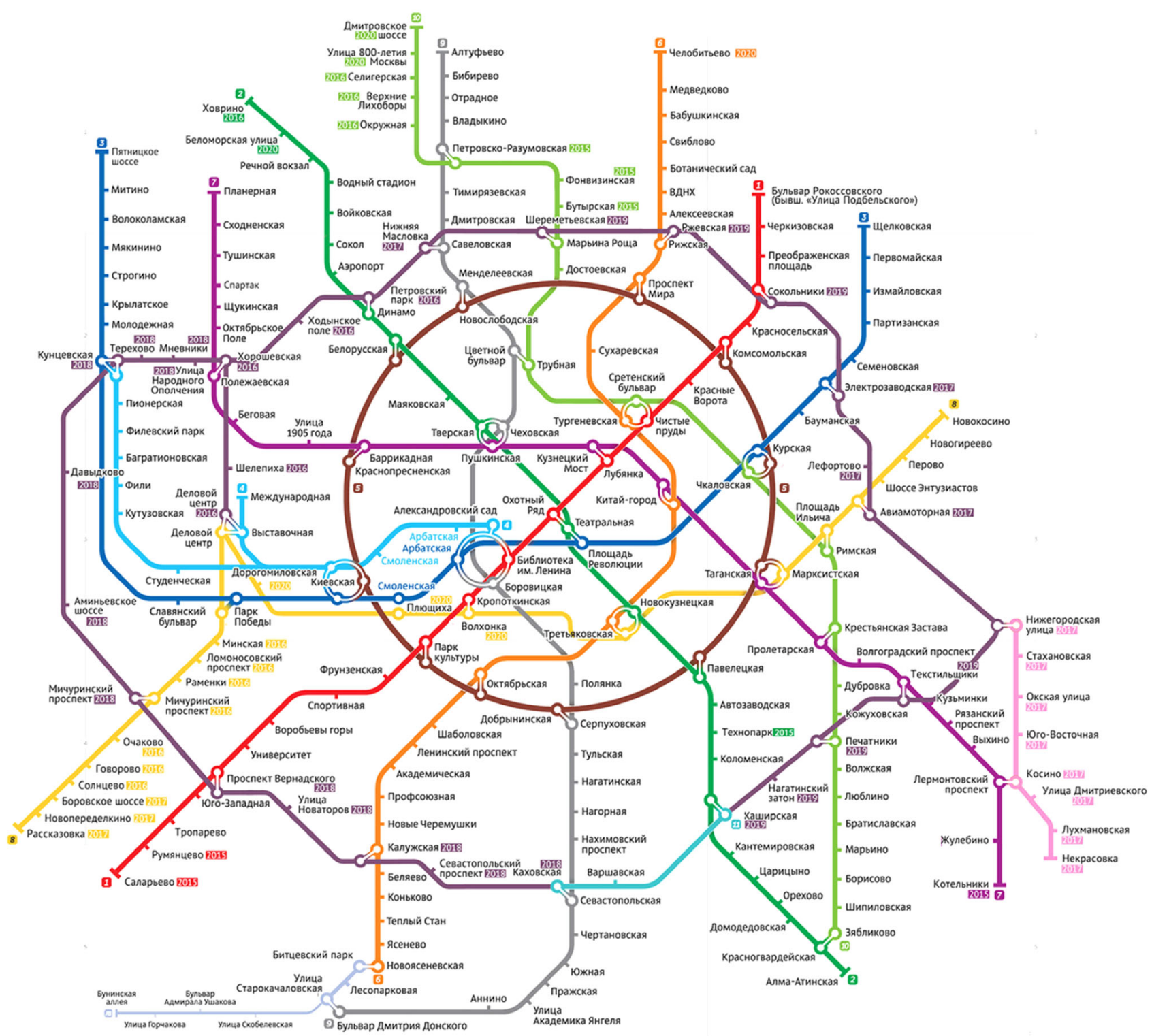

Figure 6. The extension plans for the Moscow metro. 
new point $x_{j}$ in the sought-for tuple carries a positive electric charge (so that they repulse by Coulomb's law). To prevent an escape of new points from the gamut $\Gamma$ across its boundary, put a negligibly small but positive charge at the boundary's closest point to $x_{j}$ for every $j$. By starting from random initial data $x_{j}(0) \in \Gamma$, the configurations $\left\{x_{j}(t)\right\}$ of charges evolve inside the polyhedron $\Gamma$, which, by assumption, is filled with a viscous medium so that the new points' motion slows down; sooner or later they are captured near the local minima of the potential (see [1] for details on both methods).

Finding several new colors is still not all that one needs in order to paint the new lines on a metro map. Clearly, it is the configuration of transfers from the old to new lines that must further be taken into account-to distribute the new colors between the new lines in such a way that at every transfer station, the colors of intersecting lines are maximally different.

\section{Breaking News: An Online Poll}

The 16.8-km long Kozhukhovskaya line with eight stations on it is scheduled to appear in the Moscow underground system in 2015-2016, officials say. The new line will connect the (south)east with the city centre, see Fig. 6: starting at the ocean green, that line continues along the turquoise blue toward red.

Two colors, to freely choose from, were offered to preregistered "active citizens" during an interactive poll, which was held from 20 October until 10 November 2014. Namely, black and pink were claimed as the only colors remaining from the gamut that were not yet used to paint lines on the Moscow metro map (see Figure 1). As many as 307,350 active citizens took part in the online poll, media report; $71 \%$ voted pink and 18\% preferred black, whereas $7 \%$ trusted the choice to experts (such as designers, ethnographers, or psychologists). Finally, $4 \%$ of the respondents either made their own suggestions of the new color: turquoise, olive, coral, etc.-or even proposed to make that line on the map speckled (see [12, 13]).

\section{OPEN ACCESS}

This article is distributed under the terms of the Creative Commons Attribution 4.0 International License (http:// creativecommons.org/licenses/by/4.0/), which permits unrestricted use, distribution, and reproduction in any medium, provided you give appropriate credit to the original author(s) and the source, provide a link to the Creative Commons license, and indicate if changes were made.

Johann Bernoulli Institute for Mathematics and Computer Science

Post Office Box 407

9700 AK

Groningen

The Netherlands

e-mail: simone.griffioen@hotmail.com;

simone.griffioen@student.uva.nl

\section{REFERENCES}

[1] Griffioen, S. (2014) Maximizing color difference in metro maps. http://irs.ub.rug.nl/dbi/53b525e9ac7fb.

[2] Laconte, P. Moscow: looking to the future-Mobility. http://archive. ffue.org/PDF/Moscow-PL-02-rev.

[3] London \& Partners, http://cdn.londonandpartners.com/.

[4] Luo, M. R., Cui, G., and Rigg, B. (2001) The development of the CIE 2000 colour-difference formula CIEDE2000. Color Research and Application 26, 340-350.

[5] Moscow Metro-Official Site. http://engl.mosmetro.ru/.

[6] Moscow Urban City. http://stroi.mos.ru/.

[7] Moscow.info. http://www.moscow.info/.

[8] Munroe, Randall. xkcd Color Survey Results. http://blog.xkcd. com/2010/05/03/color-survey-results/.

[9] Okabe, A., Boots, B., Sugihara, K., and Chiu, S. N. (2000) Spatial Tessellations: Concepts and Applications of Voronoi Diagrams. John Wiley and Sons, Chichester, etc.

[10] Robertson, A. R. (1990) Historical Development of CIE Recommended Color Difference Equations. Color Research and Application 15, 167-170.

[11] Rockafellar, T. (1994) Nonsmooth Optimization. Mathematical Programming: State of the Art, 248-258.

[12] Ros Business Consulting. http://www.rbc.ru/rbcfreenews/5464e5a 3cbb20f3d459fa4a9.

[13] Russian Info Agency “Novosti." http://ria.ru/moscow/20141113/ 1033155060.html.

[14] Transport for London. https://tfl.gov.uk/. 